\title{
LncRNA SRA deregulation contributes to the development of atherosclerosis by causing dysfunction of endothelial cells through repressing the expression of adipose triglyceride lipase
}

\author{
SHUGUO YANG and JINGANG SUN
}

\author{
Department of Internal Medicine, The Central Hospital of Linyi, Linyi, Shandong 276400, P.R. China
}

Received April 5, 2017; Accepted October 27, 2017

DOI: $10.3892 / \mathrm{mmr} .2018 .9497$

\begin{abstract}
It has been well established that long non-coding RNAs (lncRNAs) are crucial mediators in a diverse range of diseases, including atherosclerosis. The present study aimed to examine the molecular mechanisms underlying the association between steroid receptor RNA activator (SRA) and atherosclerosis. Reverse transcription-quantitative polymerase chain reaction analysis, western blot analysis and luciferase assays were performed to examine interactions among SRA, adipose triglyceride lipase (ATGL) and peroxisome proliferator-activated receptor (PPAR $\gamma)$, and the effect of resveratrol (RSV) on the levels of SRA, ATGL and PPAR $\gamma$. ELISA was performed to determine the effects of SRA and RSV on the production of inflammatory-associated cytokines. The results showed that knockdown of the expression of SRA by transfecting HUVECs with short hairpin RNA-SRA inhibited the production of ATGL and PPAR $\gamma$. A plasmid coding SRA RNA, but not the SRAP protein, attenuated the luciferase activity of the ATGL promoter. PPAR $\gamma$ had no effect on the luciferase activity driven by the ATGL promoter in the absence of rosiglitazone, whereas the luciferase activity of the ATGL promoter was elevated in the presence of rosiglitazone. This effect was eliminated by SRA. SRA enhanced the production of inflammatory-associated cytokines, including tumor necrosis factor- $\alpha$, interleukin-6, monocyte chemotactic protein-1 and intercellular adhesion molecule-1; however, the promoting effect of SRA was eliminated by RSV. RSV increased the expression of ATGL and PPAR $\gamma$, but not that of SRA. RSV distinctly and concentration-dependently upregulated the luciferase activity of ATGL, compared with that in the cells without RSV treatment, whereas treating with rosiglitazone inhibited the effect of RSV on the luciferase activity of ATGL. The present study examined the roles of SRA in
\end{abstract}

Correspondence to: Dr Shuguo Yang, Department of Internal Medicine, The Central Hospital of Linyi, 17 Jiankang Road, Yishui, Linyi, Shandong 276400, P.R. China

E-mail: rsvatgl@163.com

Key words: atherosclerosis, diabetes mellitus, steroid receptor RNA activator, adipose triglyceride lipase, endothelial dysfunction atherosclerosis, and the effects of changes in SRA and ATGL on inflammatory cytokines and HUVEC dysfunction.

\section{Introduction}

Atherosclerosis is the most frequent pathological process resulting in cardiovascular diseases, a disease of mediumand large-sized arteries characterized by the development of atherosclerotic plaques consisting of foam cells, leukocytes, endothelial cells (ECs), inflamed smooth muscle cells (SMCs), built-up modified lipids, calcified regions and necrotic cores (1). These characteristics of atherosclerotic plaques show that atherosclerosis is a complex disorder, and several compositions of the immune, metabolic and vascular systems are involved in this process. It is well demonstrated that diabetes mellitus is an independent risk factor of cardiovascular disease and atherosclerosis (2). Several studies have shown that patients with diabetes have higher risks of cardiovascular disease and atherosclerosis, compared with normal subjects (3).

Endothelial dysfunction (ED) has been identified as being associated with the clinical course and pathogenesis of all identified cardiovascular disorders, occurring in response to cardiovascular risk factors, followed by the occurrence of atherosclerosis $(4,5)$. ED is active in the process of lesion formation, facilitating the late and early mechanisms of atherosclerosis, and resulting in the promotion of EC permeability, vascular SMC proliferation and migration, platelet activation and oxidized-low density lipoprotein (ox-LDL), and the upregulation of leukocyte adherence, cytokine secretion, chemokine and adhesion molecules (5). For all these factors, ED is one of the main mechanisms in atherosclerotic disorders (6). The access of insulin to the tissue can be decreased by compromised endothelial functions in metabolic syndrome, and reduces the sensitivity of insulin independently from the direct effects of the muscle cells (7). The systemic knockout of adipose triglyceride lipase (ATGL), a critical enzyme in triglyceride lipolysis, leads to a murine phenotype featuring severe heart failure and progredient cardiac steatosis (8). It has been shown that global ATGL deficiency results in marked vascular endothelial dysfunction, which is partially due to the downregulated vascular expression of endothelial nitric oxide synthase (eNOS), possibly through activation/upregulation of the $26 \mathrm{~S}$ proteasome (8). 
Long non-coding RNAs (lncRNAs) are a major family that emerges from pervasive transcription, which broadly refer to RNAs $>200$ nucleotides in length with no marked ability to code. Current evidence has annotated and supported the identification of $>14,000$ lncRNA gene units in the human genome (9). Of the genome, $1 \%$ (gencode v20) comprises the exonic region of human IncRNAs, which contain approximately the same quantity of DNA as protein-coding exons (10). Equivalent, substantial quantities of IncRNA genes are estimated to present in other mammalian genomes (11). Certain lncRNAs have been shown to have a functional role in the pathogenesis of the occurrence of atherosclerosis; for example, ANRIL is closely associated with the severity of atherosclerosis (12). The H19 lncRNA is also prevalent in the neointima following damage and in human atherosclerotic lesions, but is rarely produced in normal arteries (13). Their effects in atherosclerosis, particularly in foam cells, have not been reported, although HULC is important in cell lipid afflux. Hu et al identified two lncRNAs in foam cells, namely lncRNA-DYNLRB2-2 and lncRNA-RP5-833A20.1, which modulated inflammation and foam cell cholesterol efflux (14).

It has been shown that SRA functions as a regulator of ATGL indirectly by suppressing the expression of PPAR $\gamma$, a transcription factor of ATGL, and that ATGL deficiency may cause the dysregulation of inflammatory cytokines, including tumor necrosis factor (TNF)- $\alpha$, and interleukin (IL)- $6(15,16)$. The downregulation of ATGL is responsible for the dysfunction of endothelial cells, which is one of the major causes of atherosclerosis (8). In addition, resveratrol (RSV) has been reported to suppress the inflammatory reaction caused by the deficiency of ATGL (16). In the present study, the effects of SRA and ATGL on endothelial cells were examined, and the effects of alterations of SRA and ATGL on inflammatory cytokines and endothelial cell dysfunction were investigated.

\section{Materials and methods}

Cell culture. HUVECs were obtained from Lonza (Basel, Switzerland), and radioimmunoprecipitation assay (RPMI)-1640 medium (Gibco; Thermo Fisher Scientific, Inc. Waltham, MA, USA) containing 10\% fetal bovine serum (FBS; Sijiqing Biological Engineering Materials Co. Ltd., Hangzhou, China) and $1 \%$ streptomycin-penicillin was utilized to maintain the cells under an atmosphere of $5 \% \mathrm{CO}_{2} / 95 \%$ air at $37^{\circ} \mathrm{C}$.

Vector construction. The pGL3-SRA human SRA expression vector was generated by inserting the full sequence of SRA into the pGL3-Basic vector (Promega Corporation, Madison, WI, USA) using BamHI and HindIII endonucleases, and the full sequence of SRA was amplified by PCR using DNA polymerase (Promega Corporation) and the following primer sequences: Forward, 5'-CTCCTGAAGTGGGAA ACGAAG-3'; and reverse, 5'-GAGGTTGGCTTCCATGTC TAA-3'). The thermocycling conditions used for PCR were as follows: $95^{\circ} \mathrm{C}$ for $3 \mathrm{~min}$; followed by 30 cycles of $94^{\circ} \mathrm{C}$ for $40 \mathrm{sec}, 56^{\circ} \mathrm{C}$ for $35 \mathrm{sec}$ and a final extension at $72^{\circ} \mathrm{C}$ for $60 \mathrm{sec}$. All experiments were performed three times.

Transfection. The cells were seeded into 96-well plates without antibiotics at a final density of $2 \times 10^{4}$ per well. When the
HUVECs had grown to $80 \%$ confluence, Lipofectamine ${ }^{\circledR} 2000$ (Invitrogen; Thermo Fisher Scientific, Inc.) was utilized to perform transfection. Three independent tests were run.

$R N A$ isolation and reverse transcription-quantitative polymerase chain reaction $(R T-q P C R)$ analysis. TRIzol ${ }^{\circledR}$ (Invitrogen; Thermo Fisher Scientific, Inc.) was utilized to extract total RNA from HUVECs according to the manufacturer's protocol. Subsequently, TaqMan One-Step RT-PCR master mix (Applied Biosystems; Thermo Fisher Scientific, Inc.) was used to perform RT-qPCR analysis with a $25 \mu \mathrm{l}$ reaction mixture $[2 \mu \mathrm{l}$ cDNA template $(2 \mu \mathrm{l})$, standard Taq reaction buffer $(2.5 \mu \mathrm{l}), 10 \mathrm{mM}$ dNTPs $(0.5 \mu \mathrm{l}), 10 \mu \mathrm{M}$ forward primer $\left(0.5 \mu \mathrm{l} ; 5^{\prime}\right.$-CTCCTGAAGTGGGAAACG AAG-3'), $10 \mu \mathrm{M}$ reverse primer (0.5 $\mu \mathrm{l}$; 5'-GAGGTTGGCT TCCATGTCTAA-3') and Taq DNA polymerase (1.25 $\mu \mathrm{l})$, and the remaining volume consisted of nuclease-free water]. The RT-qPCR cycling conditions were as follows: Initial polymerase activation at $95^{\circ} \mathrm{C}$ for $10 \mathrm{~min}$, followed by 40 cycles for $15 \mathrm{sec}$ at $95^{\circ} \mathrm{C}$ and $60 \mathrm{sec}$ at $60^{\circ} \mathrm{C}$. For the lncRNA SRA assay, a TaqMan RNA reverse transcription kit (Applied Biosystems, Thermo Fisher Scientific, Inc.) was used to reverse transcribe $1 \mu \mathrm{g}$ of purified RNA to single-stranded cDNA, the temperature protocol of which was performed at $16^{\circ} \mathrm{C}$ for $30 \mathrm{~min}$, followed by $42^{\circ} \mathrm{C}$ for $30 \mathrm{~min}, 85^{\circ} \mathrm{C}$ for $5 \mathrm{~min}$. Following this, qPCR was performed in 48-well plates with a Step One Plus sequence detection system (Applied Biosystems, Thermo Fisher Scientific, Inc.), and the thermocycling conditions used were as follows: $2 \mathrm{~min}$ at $50^{\circ} \mathrm{C}$ and $10 \mathrm{~min}$ at $95^{\circ} \mathrm{C}$; followed by 30 cycles of $15 \mathrm{sec}$ at $95^{\circ} \mathrm{C}$ and $60 \mathrm{sec}$ at $60^{\circ} \mathrm{C}$. U6 served as an internal control. The comparative quantification $(\mathrm{Cq})$ method (17) was utilized to calculate the relative lncRNA expression of SRA, and mRNA expression of ATGL and PPAR $\gamma$. Three independent reactions were repeated.

Luciferase assay. PCR was performed to amplify the promoter region of the ATGL human gene from the genomic DNA, and the full region of the ATGL promoter was treated with endonuclease digestion sites overhangs. The PCR-amplified fragments were then ligated to the pGL3-Basic vector (Promega Corporation) to generate the pGL3-ATGL-1LUC reporter construct. The expression vector of the human SRA pGL3-SRA expression vector was obtained by inserting full sequence of SRA into the pGL3-Basic vector (Promega Corporation). HUVECs were seeded into 96-well plates at a final density of $2 \times 10^{4}$ per well. When the HUVECs had grown to $80 \%$ confluence, Lipofectamine ${ }^{\circledR} 2000$ (Invitrogen; Thermo Fisher Scientific, Inc.) was used for co-transfection with the pGL3-SRA SRA expression vector or luciferase reporter plasmids and the pRL-TK-Renilla vector for luciferase assays. The Dual-Luciferase reporter system (Promega Corporation) was used to detect the luciferase activity of Renilla and firefly luciferase at $48 \mathrm{~h}$ post-transfection according to the manufacturer's protocol. All experiments were performed three times.

Western blot analysis. RIPA lysis buffer (Roche Diagnostics, Basel, Switzerland) was utilized to lyse HUVECs in accordance with themanufacturer's protocol.APierce bicinchoninic acidassay protein assay kit (Thermo Fisher Scientific, Inc.) was performed to quantify total protein. A $10 \%$ (w/v) polyacrylamide gel was 
A

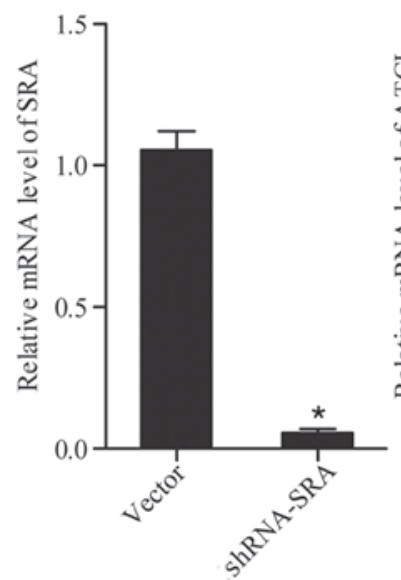

B

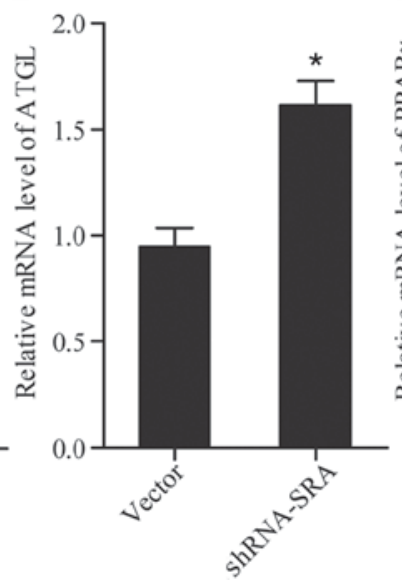

C

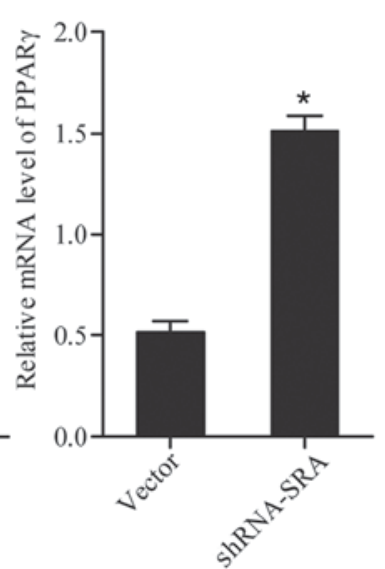

D

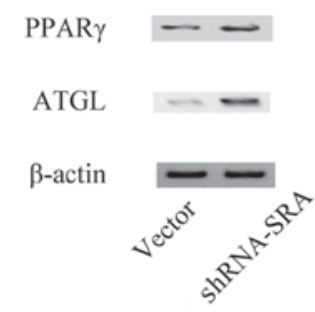

Figure 1. Effects of SRA on the expression of ATGL and PPAR $\gamma$. (A) shRNA-SRA effectively decreased the expression of SRA. (B) shRNA-SRA effectively increased the expression of ATGL. (C) shRNA-SRA effectively increased the expression of PPAR $\gamma$. (D) shRNA-SRA effectively increased the protein expression of PPAR $\gamma$ and ATGL. "P<0.01 vs. blank vector control group. shRNA, short hairpin RNA; SRA, steroid receptor RNA activator; ATGL, adipose triglyceride lipase; PPAR $\gamma$, peroxisome proliferator-activated receptor $\gamma$.

utilized to separate protein extracts with SDS-PAGE, which were then electro-transferred onto a BioTrace NT membrane (Pall Life Sciences, Port Washington, NY, USA). PBS containing $0.1 \%$ Tween-20 and 5\% fat-free milk was utilized to block the membrane for $60 \mathrm{~min}$. Primary anti-ATGL antibody at a dilution of 1:5,000 (cat. no. 3370-1; Epitomics, Burlingame CA, USA), anti-PPAR $\gamma$ antibody at a dilution of 1:5,000 (cat. no. RN075PW; Medical \& Biological Laboratories Co., Ltd., Tokyo, Japan), and anti- $\beta$-actin antibody at a dilution of 1:10,000 (cat. no. 4970; Cell Signaling Technology, Inc., Danvers, MA, USA) were utilized to maintain membrane overnight at $4^{\circ} \mathrm{C}$. Subsequently, secondary horseradish peroxidase (HRP)-labeled antibody at a dilution of 1:15,000 (cat. no. AP187P; EMD Millipore, Billerica, MA, USA) was utilized to treat the membrane at room temperature for $2 \mathrm{~h}$. Electrochemiluminescence blotting detection reagent (Thermo Fisher Scientific, Inc.) was utilized to detect the bands of the target proteins. A Chemioscope Mini system (ChemiQ 4800; Bioshine, Shanghai, China) was utilized to quantify and capture images of the protein bands of ATGL and PPAR $\gamma$. Three independent tests were performed.

ELISA. Carbonate-bicarbonate buffer $(50 \mathrm{mM})$ with mouse polyclonal IgG against ATGL (1:5,000; cat. no. 2138; Cell Signaling Technology, Inc.)/PPAR $\gamma$ (1:5,000; cat. no. sc-398394; Santa Cruz Biotechnology, Inc., Dallas, TX, USA) was utilized to treat cells $\left(2 \times 10^{3} /\right.$ well $)$ in a 24 -well plate, followed by incubation for $12 \mathrm{~h}$ at $4^{\circ} \mathrm{C}$, and PBS with $4 \%$ bovine serum albumin (BSA; Invitrogen; Thermo Fisher Scientific, Inc.) was then utilized to treat the plates for $1 \mathrm{~h}$. PBS with $0.1 \%$ Tween-20 was utilized to wash the plates twice for 3-5 min. A $50 \mu \mathrm{l}$ volume of purified intercellular adhesion molecule-1 (ICAM-1), tumor necrosis factor (TNF)- $\alpha$, IL-6 and monocyte chemotactic protein-1 (MCP-1) antigen as well as clinical samples were distributed into 96-wells, and incubated for $2 \mathrm{~h}$ at $37^{\circ} \mathrm{C}$. This was followed by washing and culture for $60 \mathrm{~min}$ at $37^{\circ} \mathrm{C}$ in $100 \mu \mathrm{l} \mathrm{PBS}$ containing $1 \% \mathrm{BSA}$ and rabbit monoclonal IgG against ICAM-1 (cat. no. 4915; Cell Signaling Technology, Inc.), TNF- $\alpha$ (cat. no. 6945; Cell Signaling Technology, Inc.), IL-6 (cat. no. 12153) and
MCP-1 (cat. no. 2027; both Cell Signaling Technology, Inc.) at dilutions of 1:1,500. Subsequently, secondary antibodies conjugated to HRP (1:3,000; cat. no. 7074; Cell Signaling Technology, Inc.) and 1\% BSA (LI-COR Biosciences, Lincoln, $\mathrm{NE}, \mathrm{USA}$ ) were utilized to maintain the plate for $24 \mathrm{~h}$ at $37^{\circ} \mathrm{C}$. A microplate reader was utilized to determine the immunoplate HRP activity with the use of $\mathrm{O}$-phenylenediamine in accordance with the absorbance at $492 \mathrm{~nm}$. $\mathrm{H}_{2} \mathrm{O}_{2}$ served as an internal control. Three independent tests were performed.

Statistical analysis. All data are presented as the mean \pm standard error of mean of three independent experiments. SPSS 23.0 statistical software (IBM SPSS, Armonk, NY, USA) was utilized to perform all statistical analysis. Student's t-test (two-tailed) was utilized to analyze differences between two groups, and the Wilcoxon signed rank test was utilized to compare relative expression levels of lncRNA SRA ATGL and PPAR $\gamma$ among groups. $\mathrm{P}<0.05$ was considered to indicate a statistically significant difference.

\section{Results}

SRA suppresses the transactivation of ATGL via PPAR $\gamma$. SRA has been shown to inhibit the transcription activity of the ATGL promoter, and it was well established that PPAR $\gamma$ serves as a transcription factor of ATGL. Therefore, the present study aimed to examine whether SRA repressed the transactivation of ATGL through PPAR $\gamma$. As shown in Fig. 1, knockdown of the expression of SRA (Fig. 1A) using shRNA against SRA substantially reduced the mRNA levels of expression ATLG (Fig. 1B) and PPAR $\gamma$ (Fig. 1C), and the protein (Fig. 1D) levels of the two. This suggested that lncRNA-SRA suppressed the production of ATGL via inhibiting the expression of PPAR $\gamma$.

SRA represses the transcription ability of the ATGL promoter via modulating PPAR $\gamma$. The endothelial cells were transfected with vectors encompassing SRA or SRAP. As shown in Fig. 2A, transfection of cells with a vector coding SRA RNA but not SRAP protein reduced the luciferase activity driven 
A

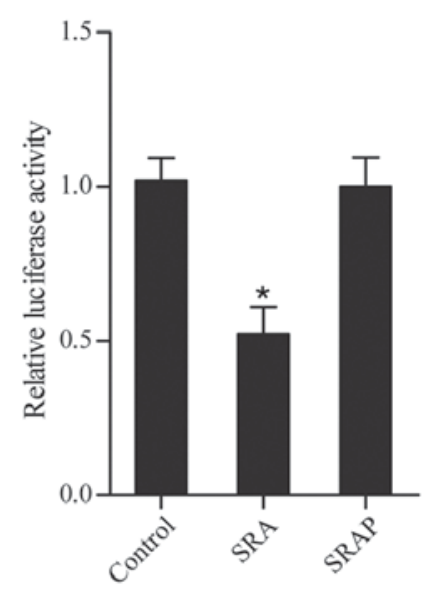

B

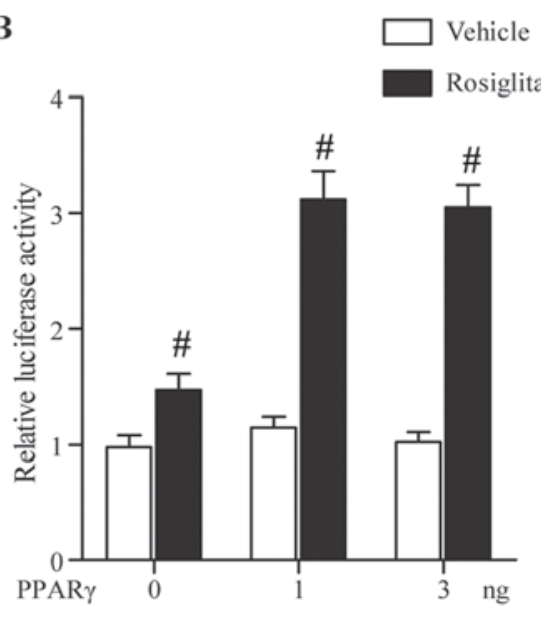

\section{C \\ zone}

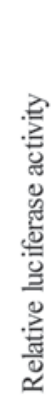

Figure 2. SRA represses the luciferase activity driven by ATGL via PPAR $\gamma$. (A) Transfection with a plasmid coding SRA RNA but not SRAP reduced the luciferase activity driven by the ATGL promoter. (B) Overexpression of PPAR $\gamma$ had no effect on luciferase activity driven by the ATGL promoter in the absence of rosiglitazone. (C) SRA eliminated the promoting effect of PPAR $\gamma$ and rosiglitazone on the activity of the ATGL promoter. ${ }^{*}<0.01$ vs. control group; ${ }^{\text {P }}<0.01$ vs. vehicle control group. SRA, steroid receptor RNA activator; SRAP, SRA protein; ATGL, adipose triglyceride lipase; PPAR $\gamma$, peroxisome proliferator-activated receptor $\gamma$.

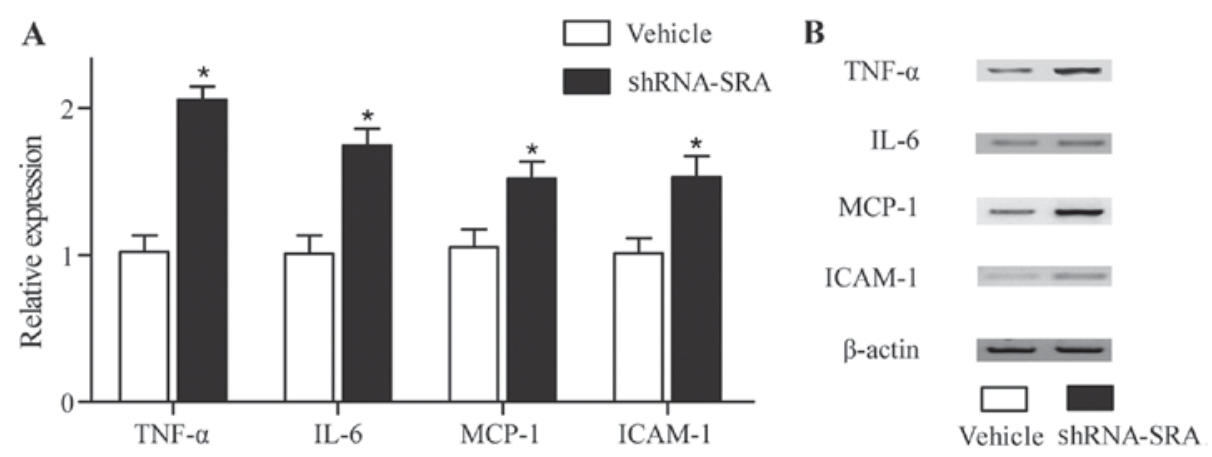

Figure 3. Repression of inflammatory-related cytokines by SRA. (A) Higher protein levels of TNF- $\alpha$, IL-6, MCP-1 and ICAM-1 were detected in HUVECs following knockdown of the expression of SRA, detected using ELISA. (B) Higher protein levels of TNF- $\alpha$, IL-6, MCP-1 and ICAM-1 were detected in HUVECs following knockdown of the expression of SRA, detected using western blot analysis. " $\mathrm{P}<0.01$ vs. vehicle control group. SRA, steroid receptor RNA activator; TNF- $\alpha$, tumor necrosis factor- $\alpha$; IL-6, interleukin-6; MCP-1, monocyte chemotactic protein-1; ICAM-1, intercellular adhesion molecule-1; shRNA, short hairpin RNA.

by the ATGL promoter, compared with control. PPAR $\gamma$ is also considered to be a transcription factor of ATGL. Therefore, it was crucial to clarify the effect of PPAR $\gamma$ on the expression of ATGL. As shown in Fig. 2B, without rosiglitazone, a PPAR $\gamma$ ligand, the upregulated expression of PPAR $\gamma$ had no effect on the luciferase activity driven by the ATGL promoter (Fig. 2B). However, in the presence of rosiglitazone, PPAR $\gamma$ increased the luciferase activity of the ATGL promoter, compared with that in the vehicle control (Fig. 2B). As shown in Fig. 2C, the promoting effect of PPAR $\gamma$ and rosiglitazone on the activity of ATGL promoter was eliminated by the overexpression of SRA (condition 3 in Fig. 2C), suggesting that SRA repressed the luciferase activity driven by ATGL via suppressing PPAR $\gamma$.

Repression of inflammatory-related cytokine levels by SRA. It is well established that inflammation is important in the development and progression of atherosclerosis, therefore ELISA and western-blot analysis were performed in the present study to investigate whether SRA was associated with atherosclerosis via affecting the production of inflammatory cytokines. As shown in Fig. 3, higher protein levels of TNF- $\alpha$, IL-6, MCP-1 and ICAM-1 (Fig. 3A and B) were observed in the HUVECs with loss of the expression of SRA, compared with control group.

$R S V$ alters the expression of SRA and downstream of SRA. RSV is known as an inhibitor of inflammation and is widely used as a drug to treat diseases caused by chronic inflammation, including atherosclerosis (18). To examine whether RSV was involved in inflammation through SRA and downstream, RT-qPCR analysis was performed to examine the levels of SRA, PPAR $\gamma$ and ATGL in cells treated with RSV. As shown in Fig. 4A, no significant difference in the levels of SRA were found between the cells treated with RSV and the untreated cells. By contrast, RSV markedly enhanced the expression of PPAR $\gamma$ (Fig. 4B) and ATGL (Fig. 4C) at the mRNA levels and protein (Fig. 4D) levels, compared with the levels in the untreated cells, confirming that RSV was involved in 

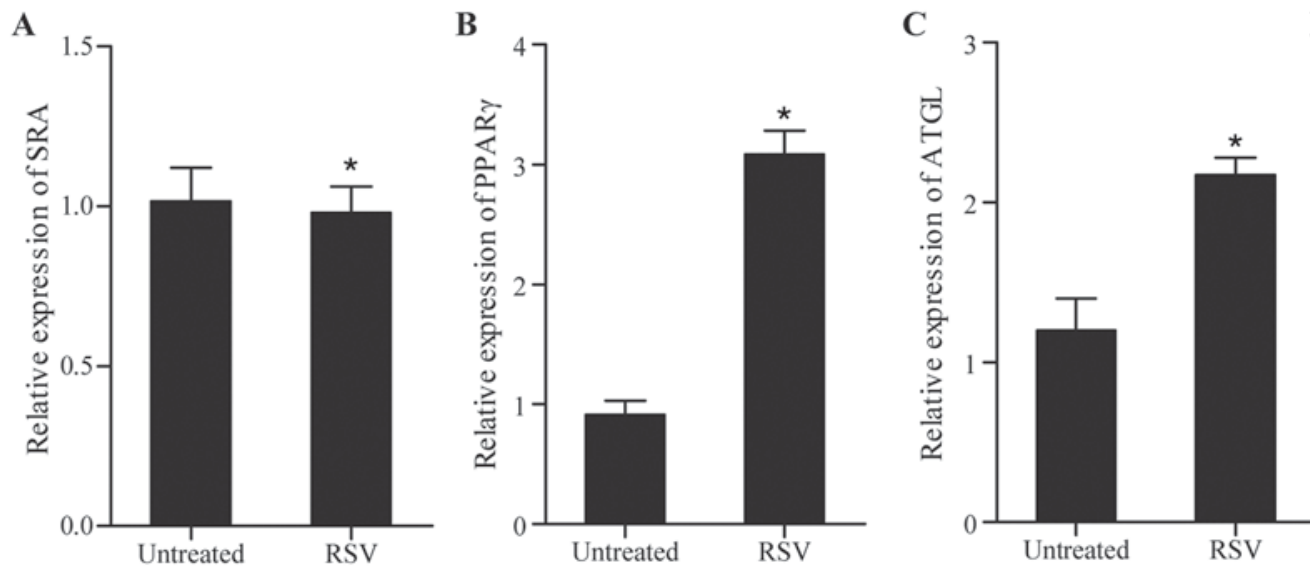

D

Figure 4. RSV alters the expression of SRA and downstream of SRA. (A) RSV suppressed the production of SRA. (B) RSV elevated the mRNA expression of PPAR $\gamma$. (C) RSV upregulated the mRNA expression of ATGL. (D) RSV increased the protein expression levels of PPAR $\gamma$ and ATGL. "P<0.01 vs. untreated group. RSV, resveratrol; SRA, steroid receptor RNA activator; ATGL, adipose triglyceride lipase; PPAR $\gamma$, peroxisome proliferator-activated receptor $\gamma$.

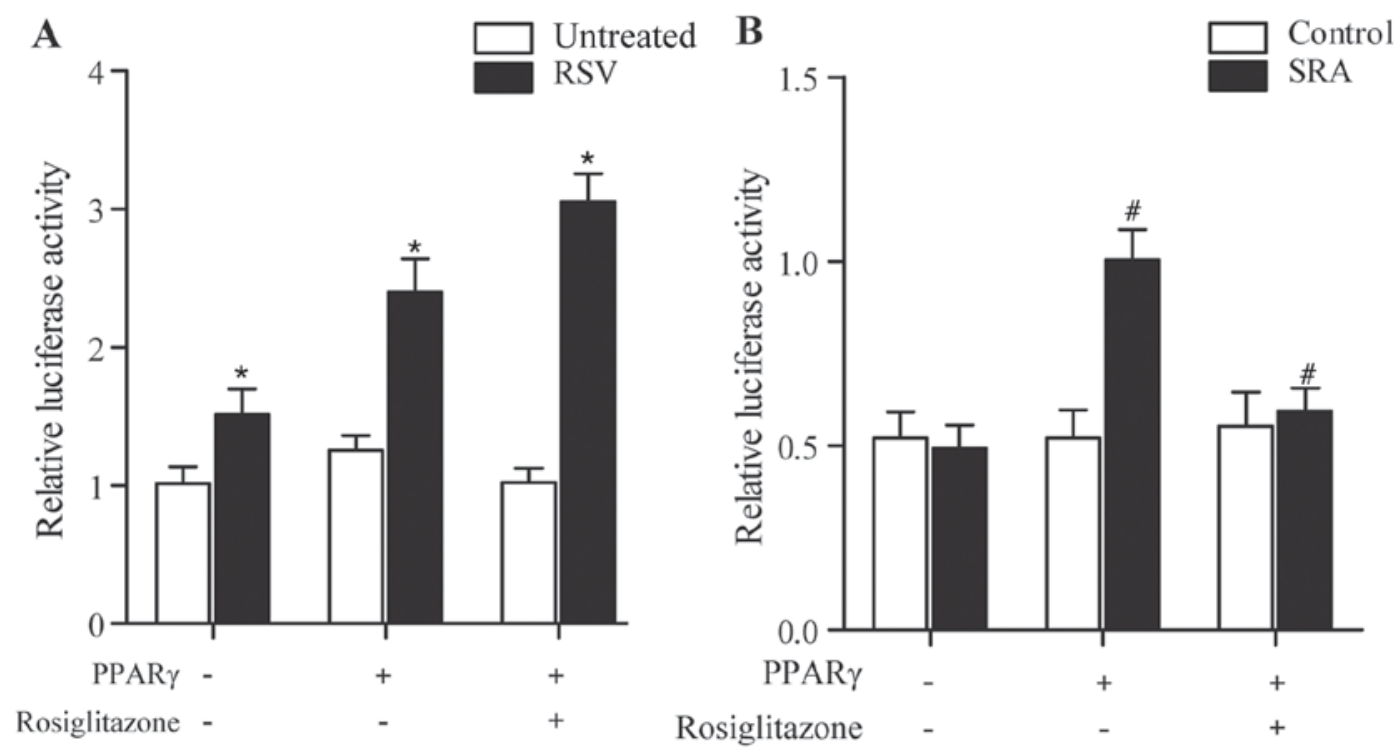

Figure 5. Effect of RSV and rosiglitazone on the luciferase activity driven by ATGL. (A) RSV markedly and dose-dependently elevated the luciferase activity driven by ATGL. (B) Rosiglitazone inhibited the effect of RSV on the luciferase activity driven by ATGL. "P<0.01 vs. untreated group; " $\mathrm{P}<0.01$ vs. control group. SRA, steroid receptor RNA activator; ATGL, adipose triglyceride lipase; RSV, resveratrol; PPAR $\gamma$, peroxisome proliferator-activated receptor $\gamma$.

inflammation through promoting the expression of PPAR $\gamma$ and ATGL.

$R S V$ represses the luciferase activity driven by ATGL. As it was found that RSV enhanced the expression of ATGL, luciferase constructs were generated to analyze this further, following which the HUVECs were treated with different doses of RSV and rosiglitazone. As shown in Fig. 5A, treatment with RSV elevated the luciferase activity driven by ATGL in a concentration-dependent manner, compared with that in the HUVECs not treated with RSV. The effect of RSV on the luciferase activity driven by ATGL was inhibited by treatment with rosiglitazone (Fig. 5B), demonstrating that RSV attenuated the luciferase activity driven by ATGL via PPAR $\gamma$. induced by SRA. ELISA and western blot analysis were performed to investigate whether RSV can eliminate the effect of SRA on the production of inflammatory-related cytokines. As shown in Fig. 6A and B, treatment with RSV eliminated the effect of treatment with SRA shRNA, suggesting that RSV eliminated the effect on the production of TNF- $\alpha$, IL-6, MCP-1 and ICAM-1 induced by SRA.

\section{Discussion}

Several lncRNAs have been reported to be involved in cardiovascular disorders. SRA, a gene encoding an lncRNA and initially identified as a steroid receptor coactivator in steroid hormone target tissues, is situated in a $600-\mathrm{kb}$ region of linkage disequilibrium associated with cardiomyopathy. Depletion of the SRA homolog in zebrafish leads to serious myocardial dysfunction (19). It has also been suggested that SRA enhances insulin-stimulated glucose uptake and 

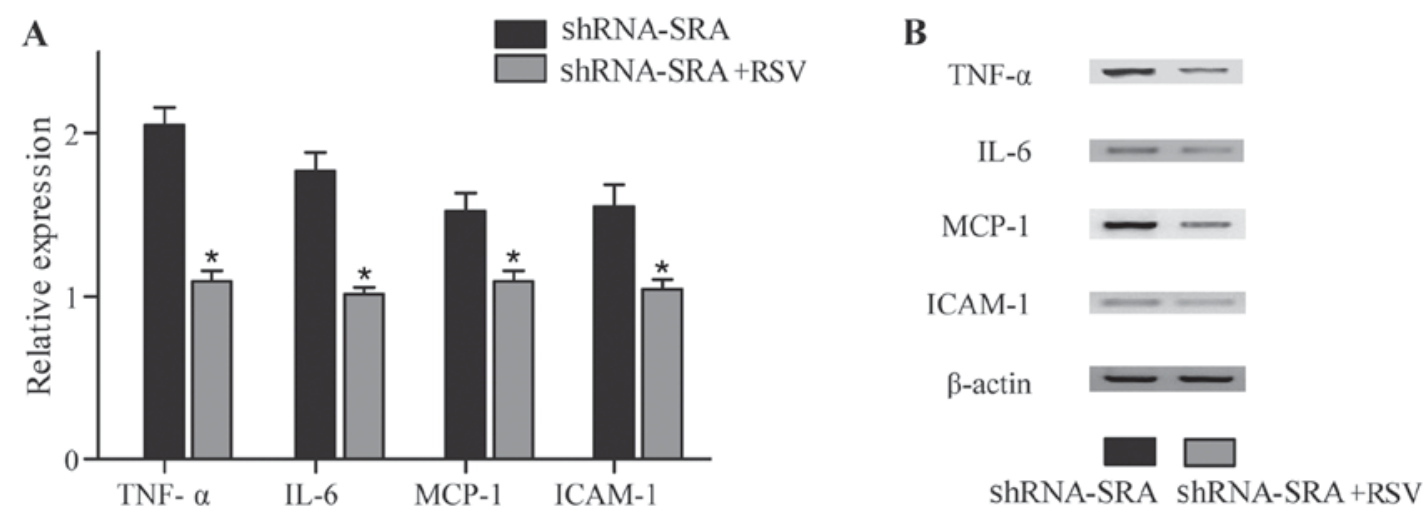

Figure 6. RSV eliminates the effect of SRA on inflammatory-related cytokines. (A) Based on the results of the ELISA, the knockdown of SRA significantly increased the production of TNF- $\alpha$, IL-6, MCP-1 and ICAM-1, whereas RSV eliminated the effects of treatment with SRA shRNA. (B) Base on results of the western blot analysis, knockdown of SRA significantly upregulated the production of TNF- $\alpha$, IL- 6 , MCP-1 and ICAM-1, whereas RSV eradicated the effect of SRA shRNA. "P<0.01 vs. shRNA-SRA group. SRA, steroid receptor RNA activator; TNF- $\alpha$, tumor necrosis factor- $\alpha$; IL-6, interleukin-6; MCP-1, monocyte chemotactic protein-1; ICAM-1, intercellular adhesion molecule-1; shRNA, short hairpin RNA; RSV, resveratrol.

adipocyte differentiation in adipocytes in vitro via multiple mechanisms, including suppressing the production of adipocyte-related inflammatory genes or enhancing the expression of insulin receptors, and coactivating the transcriptional activity of PPAR $\gamma$ (20). Previously, to evaluate the function of SRA in vivo, global Sra1 gene-knockout mice were established; these mice exhibited decreased inflammation, improved insulin sensitivity and reduced hepatic triacylglycerol contents (21). Another clinical manifestation of atherosclerosis is erectile dysfunction (ED), particularly in type II diabetes mellitus $(22,23)$. One key mechanism of ED in diabetes mellitus is a change in the signaling pathways, which results in the activation of eNOS in the endothelium. This process has been widely investigated and SRA has been found to be a regulator of this signaling pathway (24-27). The present study examined whether SRA affected the transactivation of ATGL through PPAR $\gamma$ using RT-qPCR and western blot analyses, and it was found that SRA inhibited the expression of ATGL through inhibiting the production of PPAR $\gamma$. A luciferase assay was performed to evaluate the effect of SRA on its transcription ability, and it was found that the luciferase activity of the ATGL promoter was downregulated following cell transfection with a vector coding SRA RNA.

The dysfunction of endothelial cells is considered to be important in the development of atherosclerosis, and the dysregulated production of inflammatory cytokines may be a sign of the dysfunction of endothelial cells $(28,29)$. In the present study, ELISA and western blot analysis were performed to investigate whether SRA affected the production of inflammatory cytokines, and it was revealed that SRF increased the expression of TNF- $\alpha$, IL-6, MCP-1 and ICAM-1.

ATGL was first identified as the main TG hydrolase in heart and adipose tissue. Reports indicate it also has a primary role in other tissues, including the liver (30). ATGL affects the downstream partitioning of its fatty acid (FA) products. ATGL functions as a critical modulator of PPAR in addition to its effects on FA trafficking. It has been demonstrated that mice or primary hepatocytes, which are exposed to ATGL shRNA have decreased production of PPAR $\alpha$ in addition to its downstream target genes (30). These findings are in accordance with microarray analysis of tissues in whole-body ATGL-knockout mice and previous results revealing that ATGL modulates brown adipose tissue, small intestine, and PPAR in the heart $(31,32)$.

FAs are considered the main physiological ligands to stimulate PPAR, although a number of metabolites can bind to PPAR (33). It has been shown that they are not sufficient to eliminate the chronic inflammatory changes in adipose tissue, which develop in the context of diet-induced obesity, although the suppression of ATGL-mediated adipocyte lipolysis decreases the adipose tissue inflammatory reaction to acute lipolysis (34). Of note, regardless of a comparable reduction in adipocyte lipolysis, global ATGL-knockout mice exhibit elevated, not lower, susceptibility to hepatic inflammation, likely due to the effect of hepatic ATGL being necessary for hepatic lipid homeostasis and for stimulation of the anti-inflammatory effects of PPAR $\alpha$ (35). Compared with wild-type bone marrow-transplanted LDL receptor (Ldlr)-/mice following feeding with an atherogenic Western-type diet for nine weeks, grafting of Atgl-/- bone marrow into $\gamma$-irradiated Ldlr-/- mice led to substantially reduced formation of atherosclerotic lesions. However, compromised clearance of apoptotic macrophages in advanced atherosclerotic lesions causes the formation of a necrotic core and secondary necrosis. A similar effect was found without the presence of ATGL, and TG built-up in macrophages with elevations in typical markers of apoptosis, including the externalization of phosphatidylserine in poly-(ADP-ribose) polymerase cleavage and plasma membrane, and caspase-3 (36). In the present study, it was found that RSV upregulated the luciferase activity of ATGL in a concentration-dependent manner, whereas the luciferase activity of ATGL in the cells treated with RSV and rosiglitazone was comparable with that in the untreated cell. The present study also investigated whether RSV can eliminate effect of SRA on the production of inflammatory-related cytokines using ELISA and western blot analysis, and it as revealed that RSV eliminated the promoting effect of SRA on the production of TNF- $\alpha$, IL-6, MCP-1 and ICAM-1.

In conclusion, the results of the present study indicated that lncRNA SRA promoted atherosclerosis in through repressing the expression of ATGL. The upregulated SRA suppressed the 
expression of ATGL, which induced endothelial dysfunction, and endothelial dysfunction has been established to be involved in the pathogenesis and clinical course of atherosclerosis. Therefore, lncRNA SRA may be used as a novel biomarker of atherosclerosis in patients with diabetes mellitus.

\section{Acknowledgements}

Not applicable.

\section{Funding}

No funding was received.

\section{Availability of data and materials}

The datasets used and/or analysed during the current study are available from the corresponding author on reasonable request.

\section{Authors' contributions}

SY performed the experiments, collected, analyzed and visualized the data, collected the literature and prepared the manuscript. JS designed the study, performed the experiments, analyzed the data, prepared the manuscript and approved the final manuscript.

\section{Ethics approval and consent to participate}

Not applicable.

\section{Patient consent for publication}

Not applicable.

\section{Competing interests}

The authors declare that they have no competing interests.

\section{References}

1. Ross R: Atherosclerosis-an inflammatory disease. N Engl J Med 340: 115-126, 1999.

2. Orchard TJ, Stevens LK, Forrest KY and Fuller JH: Cardiovascular disease in insulin dependent diabetes mellitus: Similar rates but different risk factors in the US compared with Europe. Int J Epidemiol 27: 976-983, 1998.

3. Himmelmann A, Hansson L, Svensson A, Harmsen P, Holmgren C and Svanborg A: Predictors of stroke in the elderly. Acta Med Scand 224: 439-443, 1988.

4. Suwaidi JA, Hamasaki S, Higano ST, Nishimura RA, Holmes DR Jr and Lerman A: Long-term follow-up of patients with mild coronary artery disease and endothelial dysfunction Circulation 101: 948-954, 2000.

5. Butany JW, Verma S, Leask RL, Mohsen B and Asa SL: Genetic abnormalities of the endothelium. Microsc Res Tech 60: 30-37, 2003.

6. Esper RJ, Nordaby RA, Vilariño JO, Paragano A, Cacharrón JL and Machado RA: Endothelial dysfunction: A comprehensive appraisal. Cardiovasc Diabetol 5: 4, 2006.

7. Kolka CM and Bergman RN: The endothelium in diabetes: Its role in insulin access and diabetic complications. Rev Endocr Metab Disord 14: 13-19, 2013.

8. Schrammel A, Mussbacher M, Wölkart G, Stessel H, Pail K, Winkler S, Schweiger M, Haemmerle G, Al Zoughbi W, Höfler G, et al: Endothelial dysfunction in adipose triglyceride lipase deficiency. Biochim Biophys Acta 1841: 906-917, 2014.
9. Derrien T, Johnson R, Bussotti G, Tanzer A, Djebali S, Tilgner H, Guernec G, Martin D, Merkel A, Knowles DG, et al: The GENCODE v7 catalog of human long noncoding RNAs: Analysis of their gene structure, evolution, and expression. Genome Res 22: 1775-1789, 2012

10. Harrow J, Frankish A, Gonzalez JM, Tapanari E, Diekhans M, Kokocinski F, Aken BL, Barrell D, Zadissa A, Searle S, et al: GENCODE: The reference human genome annotation for The ENCODE Project. Genome Res 22: 1760-1774, 2012.

11. Young RS, Marques AC, Tibbit C, Haerty W, Bassett AR, Liu JL and Ponting CP: Identification and properties of 1,119 candidate lincRNA loci in the Drosophila melanogaster genome. Genome Biol Evol 4: 427-442, 2012.

12. Holdt LM, Beutner F, Scholz M, Gielen S, Gäbel G, Bergert H, Schuler G, Thiery $\mathbf{J}$ and Teupser D: ANRIL expression is associated with atherosclerosis risk at chromosome 9p21. Arterioscler Thromb Vasc Biol 30: 620-627, 2010.

13. Han DK, Khaing ZZ, Pollock RA, Haudenschild CC and Liau G: H19, a marker of developmental transition, is reexpressed in human atherosclerotic plaques and is regulated by the insulin family of growth factors in cultured rabbit smooth muscle cells. J Clin Invest 97: 1276-1285, 1996.

14. $\mathrm{Hu} Y W$, Yang JY, Ma X, Chen ZP, Hu YR, Zhao JY, Li SF, Qiu YR, Lu JB, Wang YC, et al: A lincRNA-DYNLRB2-2/GPR119/GLP-1R/ABCA1-dependent signal transduction pathway is essential for the regulation of cholesterol homeostasis. J Lipid Res 55: 681-697, 2014.

15. Chen G, Yu D, Nian X, Liu J, Koenig RJ, Xu B and Sheng L: LncRNA SRA promotes hepatic steatosis through repressing the expression of adipose triglyceride lipase (ATGL). Sci Rep 6: 35531, 2016.

16. Lettieri Barbato D, Tatulli G, Aquilano $\mathrm{K}$ and Ciriolo MR: Inhibition of age-related cytokines production by ATGL: A mechanism linked to the anti-inflammatory effect of resveratrol. Mediators Inflamm 2014: 917698, 2014.

17. Livak KJ and Schmittgen TD: Analysis of relative gene expression data using real-time quantitative PCR and the 2(-Delta Delta C(T)) method. Methods 25: 402-408, 2001.

18. Feng Q, Su Z, Song S, Xu H, Zhang B, Yi L, Tian M and Wang H: Histone deacetylase inhibitors suppress RSV infection and alleviate virus-induced airway inflammation. Int $\mathrm{J}$ Mol Med 38: 812-822, 2016.

19. Friedrichs F, Zugck C, Rauch GJ, Ivandic B, Weichenhan D, Müller-Bardorff M, Meder B, El Mokhtari NE, Regitz-Zagrosek V, Hetzer R, et al: HBEGF, SRA1, and IK: Three cosegregating genes as determinants of cardiomyopathy. Genome Res 19: 395-403, 2009.

20. Xu B, Gerin I, Miao H, Vu-Phan D, Johnson CN, Xu R, Chen XW, Cawthorn WP, MacDougald OA and Koenig RJ: Multiple roles for the non-coding RNA SRA in regulation of adipogenesis and insulin sensitivity. PLoS One 5: e14199, 2010.

21. Liu S, Sheng L, Miao H, Saunders TL, MacDougald OA, Koenig RJ and $\mathrm{Xu}$ B: SRA gene knockout protects against diet-induced obesity and improves glucose tolerance. J Biol Chem 289: 13000-13009, 2014.

22. Creager MA, Lüscher TF, Cosentino F and Beckman JA: Diabetes and vascular disease: Pathophysiology, clinical consequences, and medical therapy: Part I. Circulation 108: 1527-1532, 2003.

23. Benjamin EJ, Larson MG, Keyes MJ, Mitchell GF, Vasan RS, Keaney JF Jr, Lehman BT, Fan S, Osypiuk E and Vita JA: Clinical correlates and heritability of flow-mediated dilation in the community: The Framingham Heart Study. Circulation 109: 613-619, 2004.

24. Tabit CE, Chung WB, Hamburg NM and Vita JA: Endothelial dysfunction in diabetes mellitus: Molecular mechanisms and clinical implications. Rev Endocr Metab Disord 11: 61-74, 2010.

25. Wheatcroft SB, Williams IL, Shah AM and Kearney MT: Pathophysiological implications of insulin resistance on vascular endothelial function. Diabet Med 20: 255-268, 2003.

26. Festa A, Hanley AJ, Tracy RP, D'Agostino R Jr and Haffner SM: Inflammation in the prediabetic state is related to increased insulin resistance rather than decreased insulin secretion. Circulation 108: 1822-1830, 2003.

27. Shoelson SE, Lee J and Yuan M: Inflammation and the IKK beta/I kappa B/NF-kappa B axis in obesity- and diet-induced insulin resistance. Int J Obes Relat Metab Disord 27 (Suppl 3): S49-S52, 2003. 
28. Sourris KC,Lyons JG, de Courten MP,Dougherty SL, Henstridge DC Cooper ME, Hage M, Dart A, Kingwell BA, Forbes JM and de Courten B: c-Jun NH2-terminal kinase activity in subcutaneous adipose tissue but not nuclear factor-kappaB activity in peripheral blood mononuclear cells is an independent determinant of insulin resistance in healthy individuals. Diabetes 58: 1259-1265, 2009.

29. Georgiou HM, Lappas M, Georgiou GM, Marita A, Bryant VJ, Hiscock R, Permezel M, Khalil Z and Rice GE: Screening for biomarkers predictive of gestational diabetes mellitus. Acta Diabetol 45: 157-165, 2008.

30. Ong KT, Mashek MT, Bu SY, Greenberg AS and Mashek DG: Adipose triglyceride lipase is a major hepatic lipase that regulates triacylglycerol turnover and fatty acid signaling and partitioning. Hepatology 53: 116-126, 2011.

31. Ahmadian M, Abbott MJ, Tang T, Hudak CS, Kim Y, Bruss M, Hellerstein MK, Lee HY, Samuel VT, Shulman GI, et al: Desnutrin/ATGL is regulated by AMPK and is required for a brown adipose phenotype. Cell Metab 13: 739-748, 2011.

32. Haemmerle G, Lass A, Zimmermann R, Gorkiewicz G, Meyer C, Rozman J, Heldmaier G, Maier R, Theussl C, Eder S, et al: Defective lipolysis and altered energy metabolism in mice lacking adipose triglyceride lipase. Science 312: 734-737, 2006.
33. Chakravarthy MV, Lodhi IJ, Yin L, Malapaka RR, Xu HE, Turk J and Semenkovich CF: Identification of a physiologically relevant endogenous ligand for PPARalpha in liver. Cell 138: 476-488, 2009.

34. Schoiswohl G, Stefanovic-Racic M, Menke MN, Wills RC, Surlow BA, Basantani MK, Sitnick MT, Cai L, Yazbeck CF, Stolz DB, et al: Impact of reduced ATGL-mediated adipocyte lipolysis on obesity-associated insulin resistance and inflammation in male mice. Endocrinology 156: 3610-3624, 2015.

35. Jha P, Claudel T, Baghdasaryan A, Mueller M, Halilbasic E, Das SK, Lass A, Zimmermann R, Zechner R, Hoefler G and Trauner M: Role of adipose triglyceride lipase (PNPLA2) in protection from hepatic inflammation in mouse models of steatohepatitis and endotoxemia. Hepatology 59: 858-869, 2014.

36. Lammers B, Chandak PG, Aflaki E, Van Puijvelde GH, Radovic B, Hildebrand RB, Meurs I, Out R, Kuiper J, Van Berkel TJ, et al: Macrophage adipose triglyceride lipase deficiency attenuates atherosclerotic lesion development in low-density lipoprotein receptor knockout mice. Arterioscler Thromb Vasc Biol 31: 67-73, 2011. 